\title{
Contact lenses and the cornea: age and length
}

\section{of wear}

\author{
R A Weale
}

\begin{abstract}
During a monitoring study related to photorefractive keratectomy, objective tests with the Oqual (a device that can be attached to the slit-lamp) were made of the image forming quality of treated, central, and untreated peripheral regions of the cornea. In a significant number of cases the untreated part was optically inferior to the treated part. Observations on 183 patients implicated prior contact lens wear: patients who had worn hard or gas permeable lenses scored less well than those wearing soft ones or none at all. The effects of age and length of wear were analysed. With one exception, corneal quality did not correlate with age or with length of contact lens wear either for the total sample (A) or for those aged 40 years and less (B). In $A$, all contact lens wearers scored less well than those who had never worn any. In $B$, whose eye lenses were most probably more transparent than those of the older group $(A-B)$, those who had worn hard or gas permeable lenses scored significantly less than soft contact lens wearers or those who had never worn any. Although visual acuity is unimpaired, the optical capacity of the affected peripheral corneal regions appears to be permanently degraded, and the observation may have a potential bearing - for example, on the choice of contact lens types selected for cosmetic reasons.

(Brf Ophthalmol 1995; 79: 163-165)
\end{abstract}

The image forming function of the anterior segment of the human eye has recently been shown to be quantifiable by objective means. ${ }^{12}$ This is achieved by means of the Oqual, a device attachable to the slit-lamp. It contains a structured image consisting of six pairs of black squares, the closest distance between a pair being $0.11 \mathrm{~mm}$, and doubling at every step. Unseen by the patient, this target is reflected by the cornea and the posterior surface of the crystalline lens, which inverts the image. The specialist's task consists in counting the number of pairs seen and resolved in the image reflected by the lens: when the anterior segment and the lens are normal, all six can be resolved. However, this number is reduced in the presence of haze or opacities or local changes in refractive index. Although the assessment can be related to the patient's visual acuity, ${ }^{2}$ a comparative study such as the present one does not require this. (It may be noted parenthetically that the double traverse of the light through the cornea and lens materially contributes to the sensitivity of the method.) Thus the resolution of six pairs of dots (that is, a score of 6.0 ) implies an optical resolving power of $6 / 4 \cdot 3(20 / 14 \cdot 3)$ and a score of 5.0 one of $6 / 6(20 / 20) .{ }^{1}$ The equivalent variation in the average scores observed in this study therefore ranges approximately across the Snellen line of $6 / 5(20 / 16)$.

The device has now been used in a quantified assessment of the effects of photorefractive keratectomy, which will be reported later. As a control test, measurements were made through the supposedly clear corneal periphery, in addition to those taken through the lesion. In a significant number of patients the control revealed a worse score than the treated area. This clearly required investigating. It should be stressed that the present report is not concerned with the effects of photorefractive keratectomy per se. The latter happened to act as a vehicle for the discovery of differential effects of various types of contact lenses on non-central parts of the cornea which could have been studied also on untreated eyes.

\section{Method}

There was no selection of patients: they were examined in the order that available time permitted in between the optical and clinical examinations of their operated eyes. In this study, they were seated at the slit-lamp, and a focusable fixation spot was provided to minimise the effects of eye movements. Oqual measurements were made first through the centre of the cornea - that is, the lesion, and then through either the left or right peripheral corneal areas about $3 \mathrm{~mm}$ off centre. In the latter case the illuminating beam entered on one side, and its reflection was observed diametrically opposite.

After the data had been recorded, the patient was asked about contact lens wear, if any. In most cases, patients were unspecific about daily routines relating to numbers of hours of wear, or the care they took of their contact lenses. However, they were fairly confident as to the number of years they had worn them when they could tolerate them, and the shorter approximate number of weeks or months when they could not tolerate them.

Records were made of the type(s) of contact lens worn - that is, hard $(\mathrm{H})$, gas permeable $(G)$, or soft (S), and the remembered duration(s) of wear.

The optical quality of the crystalline lens may diminish after the age of 40 years. This could confound conclusions drawn from the results as regards corneal transparency. Patients' ages were therefore also recorded. 
Table 1 Typical results obtained for each group of contact lens wearer

\begin{tabular}{|c|c|c|c|c|c|}
\hline \multirow{2}{*}{$\begin{array}{l}\text { Type of lens } \\
\text { and length } \\
\text { of wear in } \\
\text { years }\end{array}$} & \multirow[b]{2}{*}{ Sex/age } & \multirow{2}{*}{$\begin{array}{l}\text { Time } \\
\text { postop } \\
\text { (months) }\end{array}$} & \multirow{2}{*}{$\begin{array}{l}\text { Visual } \\
\text { acuity }\end{array}$} & \multicolumn{2}{|l|}{ Score } \\
\hline & & & & Periphery & Lesion \\
\hline $\begin{array}{l}\text { H } 9, \text { G } 6 \\
\text { H } 2, S 5 \\
\text { G } 0 \cdot 15, \text { S } 10 \\
\text { G } 11 \\
S_{0} 12 \\
0\end{array}$ & $\begin{array}{l}F / 32 \\
M / 38 \\
M / 37 \\
F / 24 \\
F / 44 \\
M / 24\end{array}$ & $\begin{array}{r}6 \\
1 \\
12 \\
1 \\
12 \\
1\end{array}$ & $\begin{array}{l}1 \cdot 2-2 \\
0 \cdot 67 \\
1 \cdot 2-3 \\
1 \cdot 2-2 \\
1 \cdot 2-1 \\
1 \cdot 2\end{array}$ & $\begin{array}{l}5 / 6 \\
4 / 6 \\
5 / 6 \\
5 / 6 \\
6 / 6 \\
6 / 6\end{array}$ & $\begin{array}{l}6 / 6 \\
4 / 6 \\
5 / 6 \\
4 / 6 \\
6 / 6 \\
5 / 6\end{array}$ \\
\hline
\end{tabular}

$\mathrm{H}=$ hard, $\mathrm{G}=$ gas permeable, $\mathrm{S}=$ soft, $0=$ none.

Table 2 Partial correlation coefficients

\begin{tabular}{|c|c|c|}
\hline & $A$ & $B$ \\
\hline \multicolumn{3}{|c|}{ (a) Measurements versus age } \\
\hline $\begin{array}{l}\mathbf{H}+\mathbf{S} \\
\mathrm{H}+\mathbf{S} \\
\mathrm{H} \\
\mathrm{S} \\
0\end{array}$ & $\begin{array}{l}-0.2732 \\
-0.2827 \\
-0.3844^{\star} \\
-0.1138 \\
-0.3035\end{array}$ & $\begin{array}{l}-0.2773 \\
-0.2361 \\
-0.0533 \\
-0.1514 \\
-0.0372\end{array}$ \\
\hline \multicolumn{3}{|c|}{ (b) Measurements versus } \\
\hline $\mathbf{H}+\mathbf{S}$ & +0.0963 & $+0 \cdot 1400$ \\
\hline $\mathbf{H}+\mathbf{S}$ & $+0 \cdot 1574$ & +0.0351 \\
\hline H & +0.1419 & -0.0662 \\
\hline$S$ & -0.0302 & -0.0261 \\
\hline & -0.3035 & -0.0372 \\
\hline
\end{tabular}

${ }^{\star} \mathrm{p}<0.005$.

$\mathrm{A}=$ all patients; $\mathrm{B}=$ those aged 40 years and less. Bold letters indicate longer duration of wear.
The data were entered on a spread sheet (LOTUS 123), and analysed as follows.

The results were classed according to the type of contact lens worn, data for hard and gas permeable ones being combined $(\mathrm{H})$ because they may exert similar mechanical effects of the type which could lead to local changes in refractive index.

Some patients had worn both hard and soft lenses $(\mathrm{H}+\mathrm{S})$, some none at all $(0)$. No distinction was made between the sexes. Averages and standard deviations of the readings were calculated for the four classes. Correlation coefficients were determined as between the measurements and age and remembered length of wear respectively. The calculations covered (A) the total sample $(n=183)$, and (B) those 40 years old and less $(n=143)$. Partial correlation coefficients were also calculated so that the effects of age and length of wear on the measurements could be isolated.

\section{Results}

It is hardly feasible, and would not be very informative, if the results for 183 cases appeared in tabular form. Raw results for a case in each group are shown in Table 1.

Two entries were made where necessary for those patients who had worn soft and other lenses in turn to allow for the different periods of wear involved. Table 2a shows the partial correlation coefficients for Oqual measurements for the corneal periphery versus age, and Table $2 \mathrm{~b}$ versus length of wear. The values of $r$ are non-significant except for hard/gas permeable lens wearers when those over 40 years old are included (A).

Table 3 Average values of the measurements (SD) [number of patients]. For details see Table 1

\begin{tabular}{lll}
\hline & $A$ & $B$ \\
\hline $\mathrm{H}+\mathrm{S}$ & $5 \cdot 16(0.7081)[38]$ & $5.23(0.7994)[26]$ \\
$\mathrm{H}$ & $5.02(0.9205)[46]$ & $5 \cdot 24(0.7692)[34]$ \\
$\mathrm{S}$ & $5.28(0.5785)[63]$ & $5 \cdot 61(0.5906)[54]$ \\
0 & $5.67(0.5774)[36]$ & $5 \cdot 72(0.5184)[29]$ \\
\hline
\end{tabular}

Table $4 \mathrm{t}$ Values and significance levels for differences between the intracolumnar averages in $A$ and $B$ in Table 2

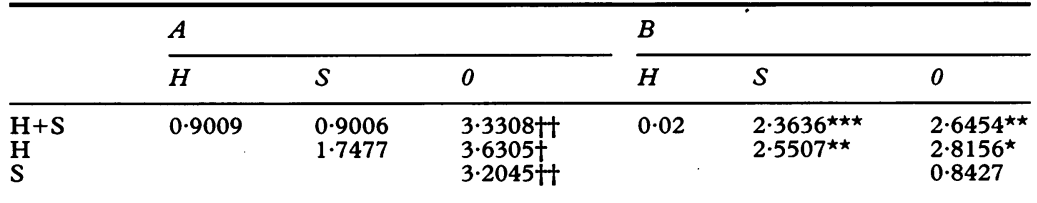

p Values: $t<0.001,+t<0.005,{ }^{\star}<0.01,{ }^{\star \star}<0.02, \star \star \star<<0.025$.
Table 3 shows average values and standard deviations of the measurements, and is subdivided into A and B as before. By and large, there is a systematic increase in the score between hard/gas permeable contact lens wearers and the others respectively. It is of interest to know whether there are any statistically significant differences between the mean values listed in this table.

Table 4 shows that this is, in fact, the case but that it differs depending on whether or not one includes the older subjects. If they are included, then the data for all types of contact lens wearer do not differ from each other but do differ from those who have never worn any. However, if one confines one's attention to those who are 40 years or less in age, the situation is very different. Those who have worn both hard and gas permeable lenses and soft ones do not differ from those who have worn both hard and gas permeable lenses but never any soft ones. In addition, those who have worn only soft lenses do not differ from those who have never worn any. The other groups differ from each other on a statistically significant level.

\section{Discussion}

The photorefractive keratectomy study, of which the present investigation formed one part, excluded all patients with even minor lenticular opacities. It is, therefore, not surprising that, with one exception, the measurements fail to show any significant correlation with age even though the testing beam of light twice traversed not only the cornea but also the eye lens.

Thus, with the exception of $\mathrm{H}$ under $\mathrm{A}$ in Table 2, the results suggest that the wear of hard and/or gas permeable contact lenses is associated with a statistically lower peripheral score, which does not appear to vary significantly with length of wear. In contrast, when age is allowed for, soft lenses do not seem to be associated with scores significantly different from those observed for non-wearers (Tables 2B and 3B).

A tentative conclusion to be drawn from the present work is that the periphery of the cornea can manifest optically detectable effects irrespective of the duration of wear, and without any measurable or perceived effect on eyesight. On a microscopic or submicroscopic level, such effects are likely to result from local structural changes. These may give rise to a partial dislocation of the corneal architecture leading to mini-chaos which manifests locally as a reduction in either transparency or random changes in refractive index.

Bergmanson and $\mathrm{Chu}^{3}$ and Bergmanson ${ }^{4}$ have studied epithelial damage in monkey and rabbit corneas after relatively robust mechanical intervention, which involved controlled trauma with epithelial erosion and oedema. A distinction was made between superficial and deep effects. Permanent corneal scarring, observed in the animal corneas, was considered to be rare in humans. Soft lenses can cause epithelial thinning without epithelial 
oedema, and it would seem to follow that, since soft lens wear could not be distinguished in the present study from no lens wear the optical phenomenon reported is likely to be associated with a disturbance of Bowman's membrane or the underlying stroma. Patients examined in the present study are unlikely to have tolerated the trauma used in the animal studies. It is noteworthy that the human corneal damage threshold is lower than that of pain sensation, ${ }^{5}$ in keeping with the present observation that the patients are unaware of any reduction in the optical quality of the cornea.

One may have to consider such effects on corneal structure - for example, in connection with options available for contact lenses worn for cosmetic purposes. It remains to be seen whether the observed phenomenon can also have other consequences even though contact lens wear is without effect on the success or otherwise of photorefractive keratectomy. ${ }^{6}$

I thank the surgeons of Moorfields Eye Hospital for kindly facilitating this investigation.

1 Weale R. The Oqual: a new device for measuring the optical quality of the anterior segment of the human eye. Exp Eye Res 1992; 55: 507-10.

2 Lobo RFJ, Weale RA. The objective clinical assessment of the quality of the anterior segment by means of the Oqual Doc Ophthalmol 1993; 83: 71-8.

3 Bergmanson JPG, Chu LW-F. Contact-lens induced epithelial injury. Am $f$ Optom Physiol Opt 1982; 59; 500-6.

4 Bergmanson JPG. Histopathological analysis of the corneal epithelium after contact lens wear. 7 Am Optom Assoc 1987; 58: $812-8$.

5 Millodot $M$, O'Leary DJ. Corneal fragility and its relationship to sensitivity. Acta Ophthalmol 1981; 59: 820-6.

6 Gimbel HV, Sun R. Effect of contact lens wear on photorefractive keratectomy. $\mathscr{F C L A O} 1993 ; 19: 217-21$ 